\title{
Análisis operacional de dos flotas industriales en la pesquería de merluza común (Merluccius gayi gayi), región centro-sur de Chile
}

\author{
Catterina Sobenes V., Gabriela Burgos P. \& Christian Díaz P. \\ Universidad Católica de la Santísima Concepción \\ Alonso de Ribera 2850, Concepción, Chile
}

RESUMEN. El trabajo tiene como objetivo analizar la actividad de dos flotas industriales (empresa A y empresa B) que operan sobre la pesquería de merluza común en la región centro-sur de Chile. Para ello, se utilizó información histórica de la operación de la flota pesquera entre 1998 y 2002. Se evaluaron las zonas de pesca o caladeros según indicadores de efectividad, costo unitario, rentabilidad y utilidad. Las embarcaciones se seleccionaron acorde a indicadores de eficiencia operacional. Estos resultados fueron contrastados con los caladeros visitados y las embarcaciones utilizadas por las empresas, durante el período de estudio. La zona de pesca más adecuada, se determinó según los índices de eficiencia para 13 caladeros de la región centro-sur de Chile. Se observa que ambas empresas han seleccionado adecuadamente los caladeros Tomé al oeste (caladero 9), Tomé al este (caladero 8) y al sur de Punta Lavapié (caladero 10), que tienen los mejores índices de eficiencia. Respecto a la selección de la embarcación más adecuada, se efectuó un análisis de jerarquización de las embarcaciones, por cada empresa, mediante el análisis de anomalías de indicadores de eficiencia. Ambas empresas seleccionaron aquellas embarcaciones que obtuvieron los mejores indicadores de eficiencia. Para el caladero 9 el estudio propone que la empresa A opere con el pesquero de alta mar PAMa3 y la empresa B con el PAMb4. Sin embargo, en el caladero 10, la empresa A debe utilizar el PAMa5 y no el PAMa3 utilizado históricamente, y la empresa B debe continuar operando con el PAMb1 y PAMb2 como lo ha hecho durante el periodo analizado.

Palabras clave: selección caladero, selección embarcaciones, indicadores eficiencia flota, Chile.

\section{Operational analysis of two industrial fleet in the Chilean hake (Merluccius gayi gayi) fishery in the southern-central region of Chile}

\begin{abstract}
This work aims at analysing the operation of two industrial fishing fleets (company A and company B) which works on the Chilean hake (Meluccis gayi gayi) fishery in the southern-central region of Chile. For this reason, it was used the historical information of the fleet operation from the southern-central region from 1998 to 2002 . The fishing grounds were assessed according to effectiveness indicators, unit cost, profitability and utility. The vessels were selected according to indicators of operational efficiency. These outcomes were contrasted with the fishing grounds and vessels employed by the companies during the study period. The most appropriate fishing ground was determined according to the efficiency indicators of 13 fishing grounds of the southern-central region. It must be said that both companies have adequately decided over the fishing ground selection, as they most frequently consider the fishing ground located between Tomé to the West (fishing ground 9), Tomé to the East (fishing ground 8) and southwards Punta Lavapié (fishing ground 10), zones with the best efficiency indicators. With regard to the selection of the best vessel, a ranking analysis of the vessels is carried out for each company by means of the analysis of the anomalies of the efficiency indicators. For the fishing ground 9, this study suggests that company A must operate with the fishing vessel PAMa3 and company B with PAMb4. Nevertheless, in the fishing zone 10, company A must use PAMa5 and not PAMa3 (which is historically being used), and company B has to continue operating with PAMb1 and PAMb2, as it has been done for the study period.
\end{abstract}

Key words: fishing ground selection, vessels selection, fleet efficiency indicators, Chile. 


\section{INTRODUCCIÓN}

Las empresas pesqueras de extracción, en la búsqueda de alcanzar mayores índices de productividad y competitividad en el mercado, son impulsadas a establecer estrategias operativas que le permitan optimizar la operación de pesca (INPESCA, 2002). Adicionalmente, el acelerado crecimiento de la población a nivel mundial, involucra un aumento en la demanda de alimento. Por ello, una de las pesquerías importantes para el consumo humano es la merluza común (Merluccius gayi gayi), que también es una fuente relevante de alimento de la zona central de Chile, donde se encuentra la mayor concentración demográfica del país (IFOP, 2000). Como consecuencia, este recurso tiene una alta demanda, y por lo tanto las empresas pesqueras buscan cumplir las respectivas cuotas de pesca asignadas de merluza común, de manera eficiente (INPESCA, 2002).

En esta industria la operación de pesca involucra muchas variables, entre éstas se encuentra la selección de zonas de pesca y embarcaciones a utilizar o también denominados Pesqueros de Alta Mar (PAM). A pesar de la importancia que tiene para las empresas pesqueras el contar con una óptima operación de pesca, se desconoce si la selección de caladeros de pesca y de embarcaciones es efectuada de manera eficiente. Generalmente, la selección de caladero es realizada por los patrones de pesca, que generalmente cuentan con información respecto a la presencia de captura en ciertas zonas. Sin embargo, no se ha evaluado, mediante indicadores cuantificables, la selección de caladeros y embarcaciones que permitan, de una manera objetiva, establecer una evaluación de la operación de la flota pesquera industrial (Burgos, 2004). Para ello, es necesario evaluar ciertos indicadores que reflejen la eficiencia de las operaciones realizadas en las labores de extracción del recurso pesquero. Al respecto, algunos estudios presentan indicadores útiles para establecer mecanismos de control o la evaluación de al- guna variable operacional en una flota pesquera industrial (Díaz, 1993; Cerda, 2001).

Con el fin de conocer si las empresas han sido eficientes en seleccionar las zonas o caladeros de pesca y las embarcaciones más adecuadas, el presente estudio consiste en evaluar las decisiones tomadas por dos empresas industriales de la región centro-sur, mediante indicadores operacionales y económicos. Para ello, se utilizó la información histórica proporcionada por el Instituto de Investigación Pesquera (INPESCA) correspondiente al período 1998-2002, de desembarque y zonas de pesca.

\section{MATERIALES Y METODOS}

\section{Área de estudio}

El área de estudio correspondió a la zona centro-sur de Chile, en la cual opera la flota arrastrera industrial en la pesquería de merluza común, entre $\operatorname{los} 32^{\circ}$ y $42^{\circ} \mathrm{S}$. En la Figura 1 se muestra

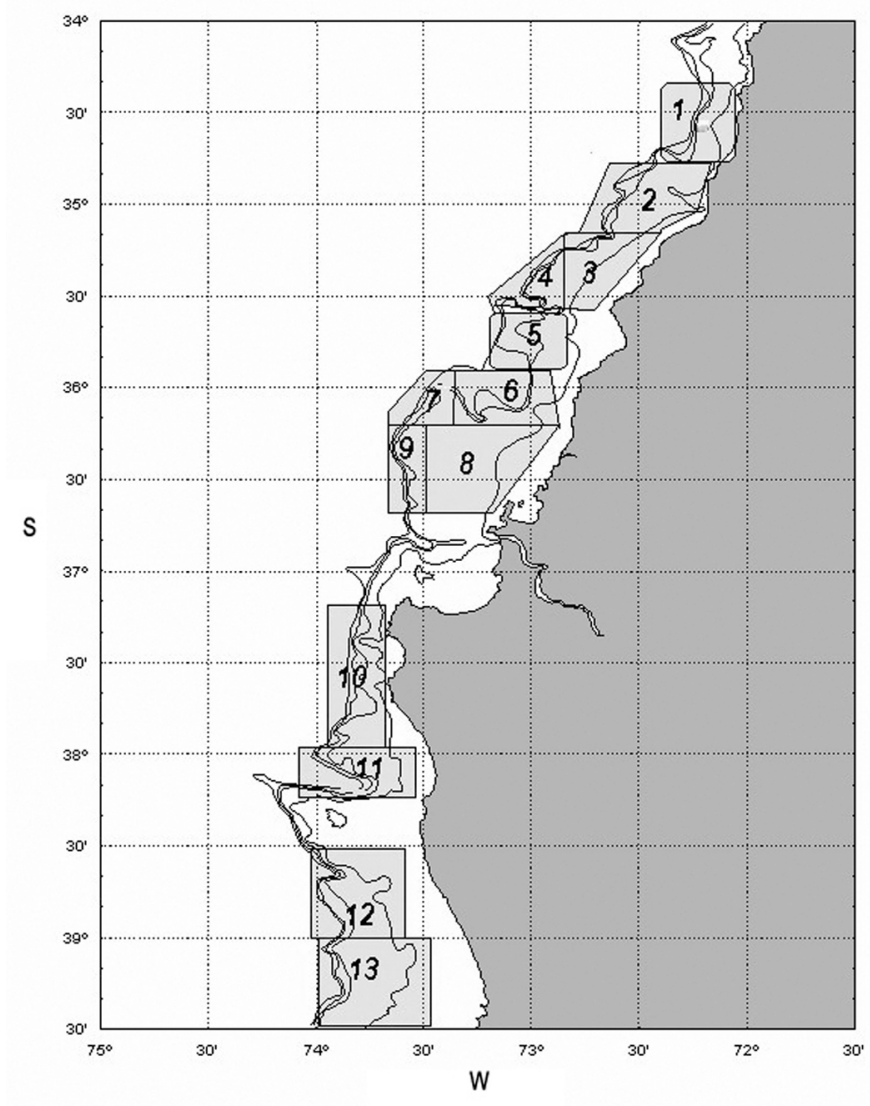

Figura 1. Zonas de pesca de merluza común (Merluccius gayi gayi) frente a la costa centro-sur de Chile (INPESCA, 2002).

Figure 1. Fishing grounds in the Chilean hake (Merluccius gayi gayi) fishery in the southern-central region of Chile (INPESCA, 2002). 
la distribución de las zonas de pesca y sus respectivos nombres en la Tabla 1 (INPESCA, 2002; Gatica \& Cubillos, 2004).

\section{Base de datos}

La información proviene de registros operacionales, de las embarcaciones industriales de dos empresas pesqueras industriales de extracción de la región centro-sur, dedicadas a la pesca de merluza común (Merluccius gayi gayi), que operaron entre 1998 y 2002. Esta información fue obtenida de datos históricos, proporcionados por el INPESCA y las empresas pesqueras involucradas en el estudio, denominadas empresas A y B. Se identificó el nombre de la embarcación (para la empresa A: PAMa y para la empresa B: PAMb), número de lances, latitud y longitud inicial y final del lance, hora de inicio y término del lance, velocidad de navegación, rumbo, peso y número de cajas con merluza común destinadas a producción (Rebolledo et al., 2002). Adicionalmente, se recopiló información económica y operacional desde las empresas pesqueras a través de entrevistas personales semi estructuradas aplicadas a los jefes de flota y personal administrativo de las empresas (Burgos, 2004). La información de costos y otros antecedentes se obtuvo de las bitácoras de pesca.

Tabla 1. Caladeros de pesca de merluza común (Merluccius gayi gayi).

Table 1. Fishing grounds of Chilean hake (Merluccius gayi gayi).

\begin{tabular}{cc}
\hline Zona & Nombre de la zona \\
\hline 1 & Punta Lobos - Punta Boyeruca \\
2 & Punta Boyeruca - Constitución \\
3 & Constitución - Carranza \\
4 & Carranza - Oeste \\
5 & Carranza - Nugurne \\
6 & Nugurne - Este \\
7 & Nugurne - Oeste \\
8 & Tomé - Este \\
9 & Tomé - Oeste \\
10 & sur de Punta Lavapié \\
11 & norte de isla Mocha \\
12 & sur de isla Mocha \\
13 & Punta Nihue \\
\hline
\end{tabular}

\section{Selección de indicadores}

Para la selección de los caladeros de pesca se definieron cuatro indicadores relacionados a: criterios de efectividad, costo unitario, rentabilidad y utilidad (Olivares, 1977). Los indicadores, se calcularon considerando tanto el valor del precio de venta por tonelada de merluza común, como los costos asociados en Unidades de Fomento (UF), valor correspondiente en pesos a noviembre de cada año (Tabla 2).

Para la estimación de costos, se estableció para cada embarcación que operó en cada empresa por zona y año, los costos fijos, costos por víveres, bonos de pesca, costo del arte y costo por combustible, obteniendo un valor de costo por viaje de pesca. Los costos fijos y víveres se estimaron, considerando la información recopilada en las empresas. Así mismo, los bonos de pesca se estimaron, según información del criterio de bonificación por tonelada capturada, para cada tripulante en cada embarcación. Por último, el costo de combustible se calculó, considerando el rendimiento del motor (litros consumido combustible por distancia recorrida) y los datos de distancias recorridas en cada viaje por cada embarcación.

Tabla 2. Indicadores para la selección de zonas o caladeros de pesca.

Table 2. Indicators for fishing ground selection.

\begin{tabular}{lc}
\hline Índice & Fórmula \\
\hline Efectividad & $\frac{A \cdot B}{C+D}$ \\
\hline Costo unitario & $\frac{E \cdot(D+C)+F+A \cdot G}{A}$ \\
\hline Rentabilidad & $\frac{(A \cdot B)}{[E \cdot(D+C)+F+A \cdot G]}$ \\
\hline Utilidad & {$[A \cdot(B-G)]-[E \cdot(D+C)+F]$} \\
\hline
\end{tabular}

donde $=$ A: captura por zona (ton); B: precio de venta por tonelada (Unidad de Fomento, UF); C: tiempo efectivo de pesca (h); D: tiempo no efectivo (tiempo de navegación más tiempo por efecto de reducción meteorológica en horas); E: costo total del viaje por hora (Unidad de Fomento, UF); F: costo total del arte (Unidad de Fomento, UF); G: bonos de pesca por tonelada de captura (Unidad de Fomento, UF). 
El indicador de efectividad, expresa el ingreso bruto obtenido por el tiempo total de operación. Mientras mayor es el valor del indicador, mayor es la efectividad de la zona de caladero. Para los tiempos considerados, se determinó el tiempo utilizado en la operación de pesca, tiempo de navegación y la reducción meteorológica causada por el mal tiempo, siendo este último un $10 \%$ del tiempo efectivo de pesca en los meses de invierno (junio a septiembre).

El costo unitario, señala los costos operacionales incurridos por cada tonelada de pesca obtenida. En consecuencia, un menor indicador de costo unitario, presenta una mejor condición de la zona de caladero.

El indicador de rentabilidad, expresa la ganancia bruta por cada unidad de costo incurrida en la operación. Luego, la zona de caladero será más rentable si el valor de este indicador corresponde al mayor valor observado.

Por último el indicador más completo, debido a que considera todos los factores, es el de utilidad, siendo la mejor zona de pesca aquella con el mayor valor de utilidad.

Todos los indicadores señalados, se analizan para cada zona, evaluando un único indicador que representa el período 1998-2002.

En relación al análisis de eficiencia de las embarcaciones que operaron en el período analizado, se evaluaron siete indicadores de comportamiento (Tabla 3) (Olivares, 1977; Díaz, 1993; Cerda, 2001; Burgos, 2004). Estos permiten medir la eficiencia en la operación, conforme a sus características operacionales y funcionales, observadas en el período de estudio. Se construyeron indicadores para cada embarcación, por zona de pesca, en el período considerado.

El indicador de comportamiento de captura por lance (CLAN), representa la eficiencia de la embarcación, al realizar el lance de pesca (Tabla 3). Así mismo, la eficiencia de captura (ECAP) expresa la eficiencia de la embarcación en término de los días efectivos con pesca del total de días en operación (Díaz, 1993).

El promedio de capturas respecto a los días con pesca, es el indicador CDP, que puede ser considerado como un índice de captura por unidad de esfuerzo.

Otro indicador de comportamiento, es el promedio bruto total (PBT), que representa el número de
Tabla 3. Indicadores de comportamiento de las embarcaciones pesqueras.

Table 3. Indicators for fishing vessel behavior.

\begin{tabular}{|c|c|}
\hline Indicador & Fórmula \\
\hline \multirow[t]{2}{*}{ CLAN } & Captura \\
\hline & Lances totales \\
\hline \multirow[t]{2}{*}{ ECAP } & Días con pesca \\
\hline & Días en el mar \\
\hline \multirow[t]{2}{*}{ PBT } & Captura \\
\hline & $\overline{\text { Capacidad de bodega }}$ \\
\hline \multirow[t]{2}{*}{ FUB } & Captura $\cdot 100$ \\
\hline & $\overline{\text { Días en el mar } \cdot \text { Capacidad de bodega }}$ \\
\hline \multirow[t]{2}{*}{$\mathrm{CDM}$} & Captura \\
\hline & Días en el mar \\
\hline \multirow[t]{2}{*}{ CAPCON } & Capturas \\
\hline & Consumo de combustible \\
\hline \multirow[t]{2}{*}{ CONCOM } & Costo consumo de combustible \\
\hline & Capturas \\
\hline
\end{tabular}

veces que la bodega de la embarcación se llenó (Díaz, 1993; Cerda, 2001). Similar al índice anterior, la eficiencia de utilización de la bodega (FUB) expresa el porcentaje de la bodega utilizado por día en el mar. Este indicador es conocido también como capacidad de acarreo.

Finalmente, se presentan los indicadores de rendimiento CAPCON y CONCOM, que reflejan la captura obtenida (en ton) por cada litro de combustible gastado, o los litros de combustible gastado por cada tonelada capturada, respectivamente (Tabla 3$)$.

\section{Jerarquización y comparación de las embarcaciones}

Después de evaluar los indicadores de comportamiento para cada embarcación pesquera por zona, de manera anual en el período 1998-2002, se procede a explorar si la flota analizada presenta distintas categorías de embarcaciones pesqueras. Para ello, se aplica el test F de Fisher (Canavos, 1988) de comparación entre las medias para distintos tratamientos, a un indicador de comportamiento de las distintas embarcaciones. El índice seleccionado corres- 
ponde a la captura por lance, dado que es considerado como el que expresa de mejor manera la eficiencia de la unidad de pesca (Salas et al., 1985).

Si la flota no se agrega, formando categorías, se debe calcular los valores promedios de cada índice de comportamiento para la flota en su conjunto, a partir de la siguiente expresión:

$$
\overline{I N D}_{\text {flota }}=\frac{\sum_{i=1}^{n} I N D_{i}}{n}
$$

donde:

$$
\begin{aligned}
\overline{I N D}_{\text {flota }}= & \text { indicador promedio de la flota } \\
I N D_{i}= & \text { indicador observado para la embarca- } \\
& \text { ción } \mathrm{i} \\
= & \text { número de embarcaciones de la flota } \\
& \text { que operó }
\end{aligned}
$$

Posteriormente, se calculan las anomalías $\left(\mathrm{ANOM}_{\mathrm{i}}\right)$ para cada índice por embarcación i:

$$
A N O M_{i}=I N D_{i}-\overline{I N D}_{\text {flota }}
$$

Para definir un índice general de comparación, es necesario asociarlo a un costo de operación relevante, donde su anomalía con signo negativo indique mayor eficiencia. Por el contrario, las anomalías de los índices de comportamiento, deben ser valores positivos, los que reflejen mayor eficiencia. Esto se debe al tipo de gráfica utilizada en el análisis presentado (Díaz, 1993; Cerda, 2001; Burgos, 2004). Por lo anterior, se ha seleccionado como índice general al indicador CONCOM.

De esta forma, los valores obtenidos por índice para cada embarcación, junto al índice general de comparación, se grafican en un plano cartesiano para obtener un ordenamiento grupal de la flota (Díaz, 1993; Cerda, 2001). Con el objetivo de jerarquizar de mayor a menor las distintas embarcaciones que componen la flota, respecto a cada uno de los índices considerados, se asigna un lugar de jerarquización a cada uno de los cuadrantes del plano cartesiano.

Los criterios de ordenamiento de las embarcaciones en los distintos cuadrantes son (Díaz, 1993):

1) La ubicación de cada embarcación en los distintos cuadrantes del plano cartesiano de control, depende de la magnitud del par ordenado en cuestión. Este par ordenado (x, y) está dado por la magnitud del índice general de comparación (x) versus la magnitud del índice de comportamiento (y) considerado.

2) Si una embarcación posee anomalía negativa en el índice general de comparación, se ubicará en los cuadrantes 1 ó 3. Si el índice de comportamiento presenta anomalía positiva, la embarcación se ubicará en el cuadrante 1 , en caso contrario se ubicará en el cuadrante 3 .

3) Si una embarcación posee anomalía positiva del índice general de comparación, se ubicará en los cuadrantes 2 ó 4. Si el índice de comportamiento presenta anomalía positiva, la embarcación se ubicará en el cuadrante 2 , en caso contrario en el cuadrante 4.

4) Las embarcaciones más eficientes se ubicarán en el cuadrante superior izquierdo (1), ya que en éste se tendrá una anomalía negativa del índice general de comparación y una anomalía positiva del índice de comportamiento (bajo costo y alta eficiencia relativa a un criterio de eficiencia determinada).

5) Posteriormente, se grafican los valores de las anomalías de los índices señalados anteriormente, para cada embarcación por zona en un plano cartesiano, obteniendo las relaciones CLANCONCOM, ECAP-CONCOM, PBT-CONCOM, CDP-CONCOM.

6) Por último, para jerarquizar la flota se evalúa el cuadrante en el cual se ubica cada embarcación, para cada uno de los índices de comportamiento considerados y por cada zona analizada. A continuación, se obtiene el promedio por embarcación de los valores de los cuadrantes obtenidos para cada índice y se ordenan de mayor a menor eficiencia.

\section{RESULTADOS}

Características de la operación de pesca de las empresas A y B durante el período 19982002

\section{Capacidad de bodega de las empresas}

La empresa A cuenta con embarcaciones con capacidad de bodega que varían entre 80 y $564 \mathrm{~m}^{3}$ correspondientes al PAMa2 y al PAMa3 respectivamente, con una capacidad media de $348 \mathrm{~m}^{3}$ y un coeficiente de variación de 0,6. En la empresa B el rango está entre 212 y $567 \mathrm{~m}^{3}$, correspondientes al 
PAMb5 y el PAMb4 respectivamente, una media de $434 \mathrm{~m}^{3}$ y un coeficiente de variación de 0,3 , lo que indica que esta última empresa presenta embarcaciones con menor variabilidad en sus bodegas (Fig. 2).

\section{Zonas de pesca más visitadas}

La empresa A durante 1998-2002 visitó en más oportunidades la zona 9, con una media de 1.359 días de navegación en esta zona y un coeficiente de variación de 0,8. La empresa $\mathrm{B}$ visitó en más oportunidades la zona 10, con una media de 926 días de navegación y un coeficiente de variación de 0,4 lo que indica que ésta última empresa presenta menor variabilidad en cuanto a los días de pesca registrados en zona (Fig. 3).

\section{Captura por unidad de área (CPUA)}

En la empresa A se observa que la zona de mayor frecuencia de visita coincide con la que obtuvo mayor CPUA (zona de pesca 9), con media de 43.245 ton $\cdot \mathrm{mn}^{-2}$ durante los cinco años en estudio y un coeficiente de variación de 0,8. En la empresa B, al igual que el caso anterior, es la zona de pesca 9 la de mayor CPUA, en esta oportunidad la media fue de 29.637 ton $\cdot \mathrm{mn}^{-2}$ y el coeficiente de variación de 0,7 . Ambas presentan alta variabilidad en cuanto a la captura por unidad de área registrada en esta zona (Fig. 4).

Captura por unidad de esfuerzo (CPUE)

La empresa A visitó con más frecuencia la zona de pesca 9, que además registra mayor CPUA y CPUE, observándose una media durante el período analizado (cinco años) de 1.602 ton.(h.a) ${ }^{-1}$ y un coeficiente de variación de 0,7 . En la empresa B la mayor CPUE durante los cinco años se observó, tanto en la zona de pesca 9 (entre los años 2000-2001) con media de 687 ton.(h.a) ${ }^{-1}$ y coeficiente de variación de 1,4 como en el caladero 10 (entre 1998 y 2002), siendo esta última zona la más visitada por esta empresa con una media de 648 ton.(h.a $)^{-1}$ y un coeficiente de variación de 0,4. Esta zona tiene menor variabilidad en cuanto a la captura por unidad de esfuerzo observada durante el período en estudio (Fig. 5).

\section{Embarcaciones de uso más frecuente por zona}

Se realizó un análisis en cada empresa de las embarcaciones que fueron utilizadas con mayor frecuencia en cada zona de pesca, las que se denominaron como " 1 a Prioridad" y aquellas embarcaciones que fueron consideradas como una segunda alternativa de uso, se les denominó " 2 a Prioridad" (Tabla 4).

\section{Determinación de las zonas de pesca y embarcaciones más convenientes a utilizar por las empresas pesqueras analizadas, según los indicadores de eficiencia utilizados}

\section{Determinación de zonas de pesca más eficientes}

Al evaluar los indicadores de eficiencia por empresa, se obtuvo un valor para cada zona analizada. Luego, fueron ordenados de manera descendente

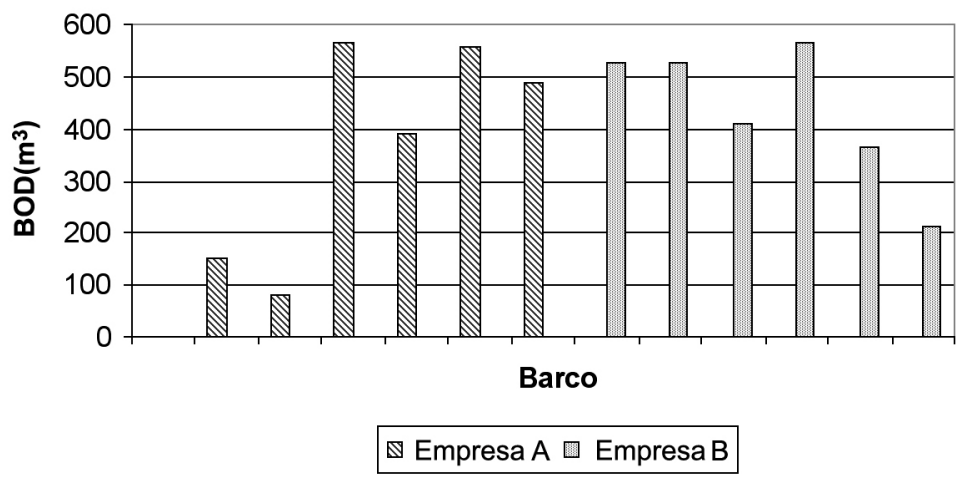

Figura 2. Capacidad de bodega de las embarcaciones de las empresas A y B que operaron en el período 19982002.

Figure 2. Fishing vessel operating storage capacity of companies A and B operating in the period 1998-2002. 

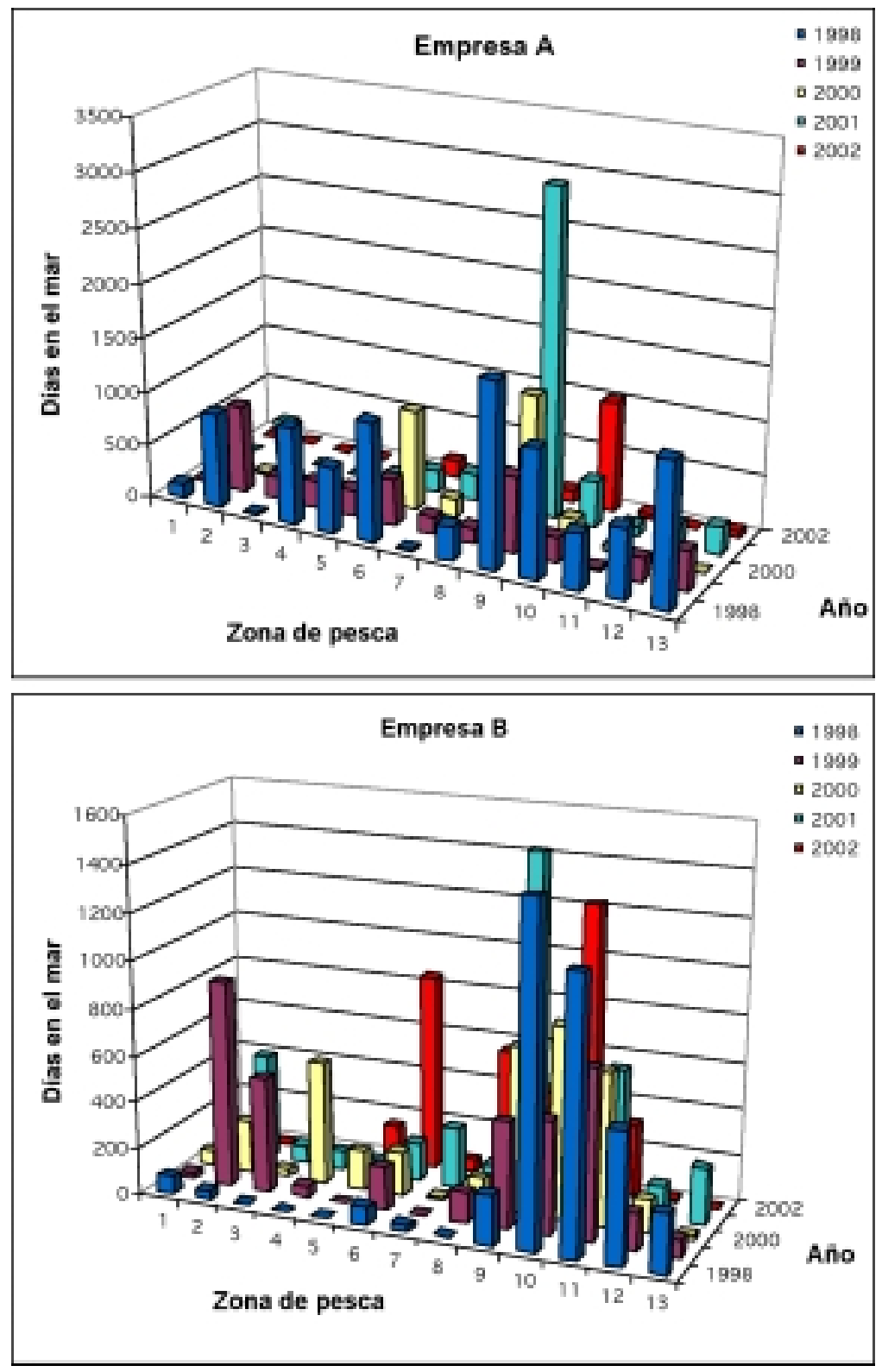

Figura 3. Zonas de pesca más visitadas por las embarcaciones de las empresas A y B, por zona y año. Figure 3. Fishing ground most visited by companies $A$ and $B$ at each zone and year. 


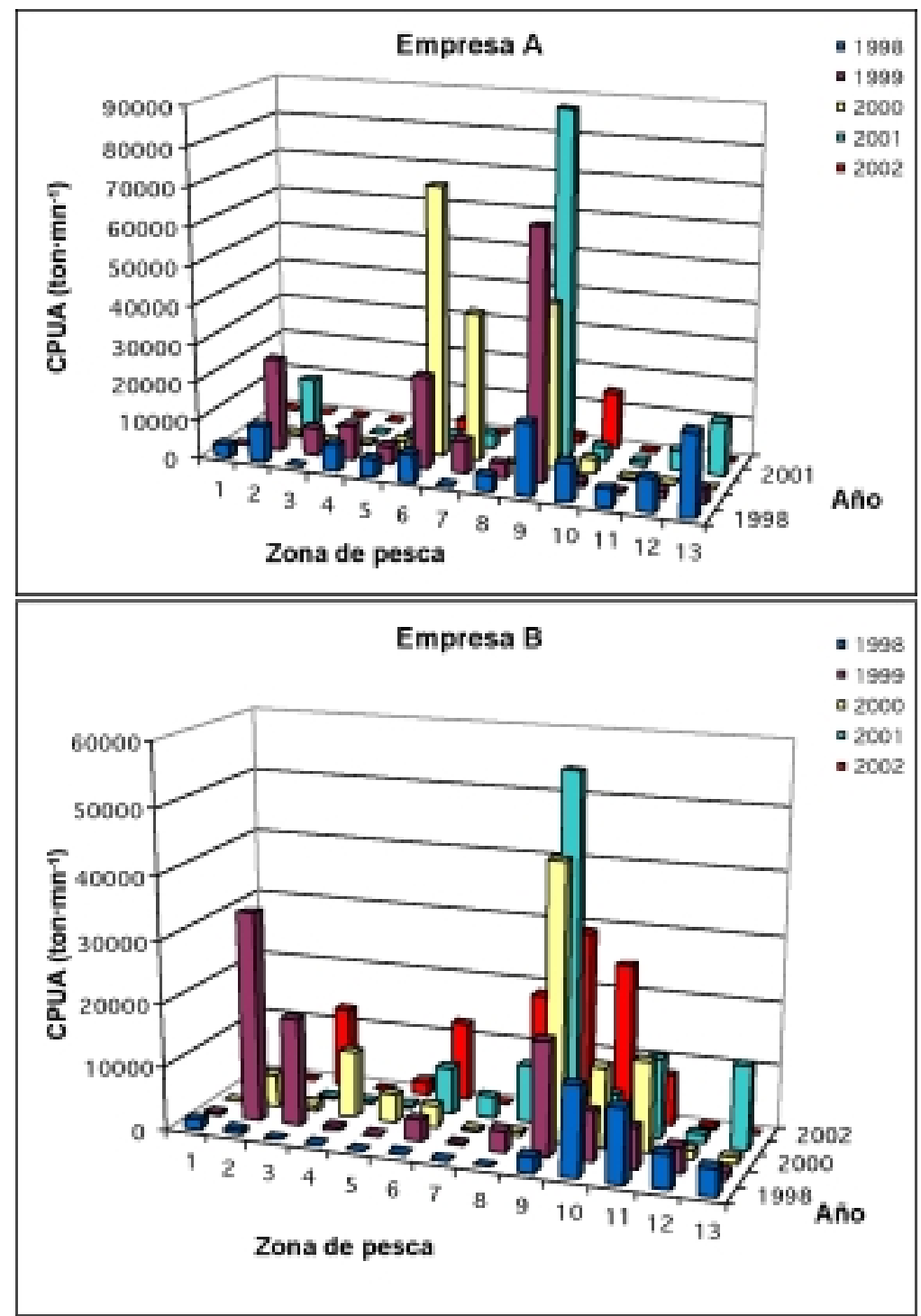

Figura 4. CPUA de las empresas A y B por zona y año.

Figure 4. CPUA of companies A and B by zone and year. 

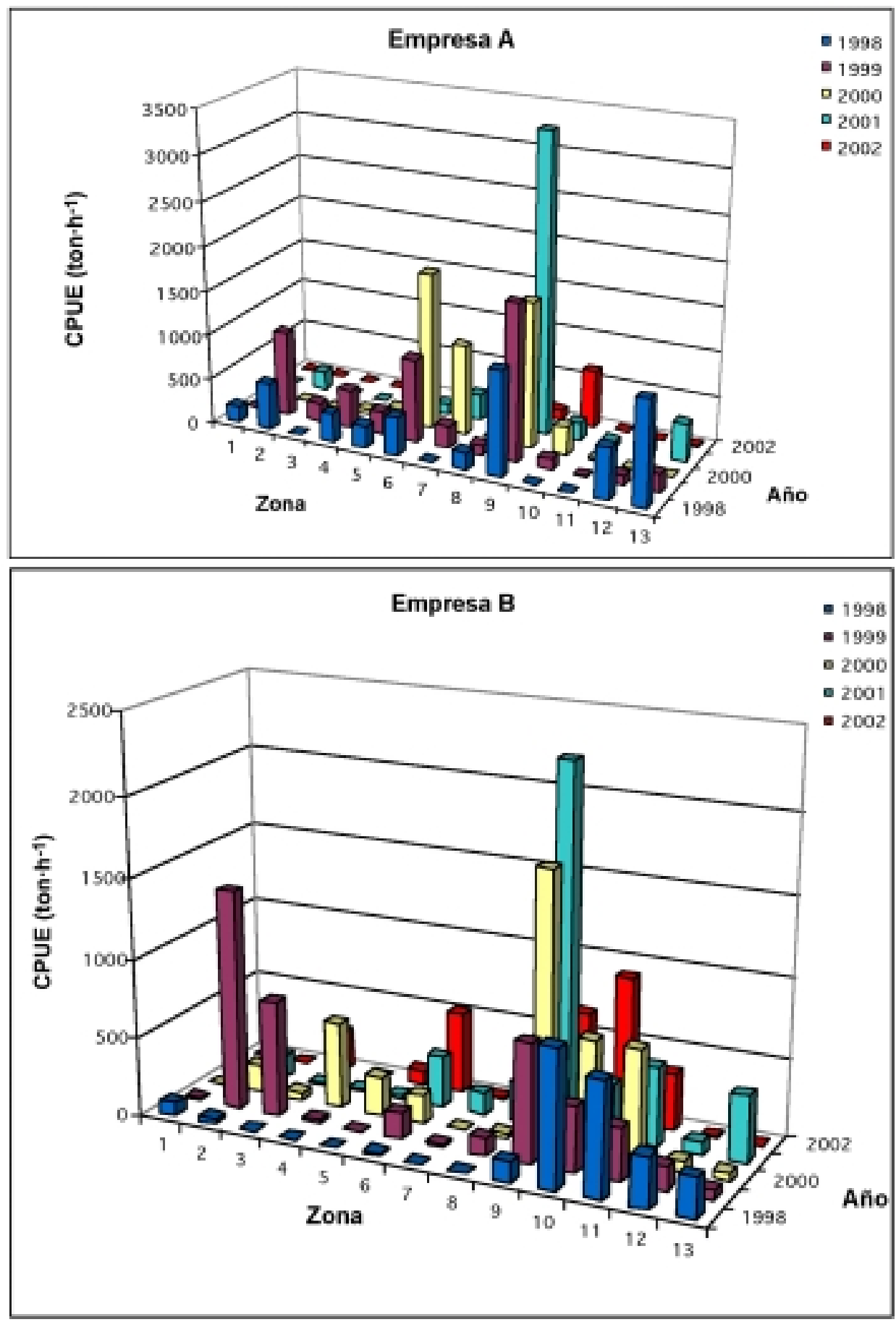

Figura 5. CPUE de las empresas A y B por zona de pesca y año.

Figure 5. CPUE of companies $A$ and $B$ by fishing zone and year. 
Tabla 4. Embarcaciones utilizadas más frecuentemente por zona.

Table 4. Vessels most frequently used by zone.

\begin{tabular}{|c|c|c|c|c|}
\hline \multirow{2}{*}{$\begin{array}{c}\text { Empresa } \\
\text { Zona }\end{array}$} & \multicolumn{2}{|c|}{ Empresa A } & \multicolumn{2}{|c|}{ Empresa B } \\
\hline & $\mathbf{1}^{\mathrm{a}}$ Prioridad & $2^{\mathrm{a}}$ Prioridad & $1^{a}$ Prioridad & $2^{\mathrm{a}}$ Prioridad \\
\hline 1 & PAMa3 & & PAMb3 & PAMb2 \\
\hline \multirow[t]{2}{*}{2} & PAMa3 & & PAMb1 & PAMb2 \\
\hline & PAMa4 & & PAMb3 & PAMb6 \\
\hline \multirow[t]{2}{*}{3} & PAMa3 & & PAMb2 & PAMb1 \\
\hline & PAMa6 & & PAMb3 & \\
\hline 4 & PAMa3 & & PAMb2 & PAMb3 \\
\hline \multirow[t]{2}{*}{5} & PAMa3 & PAMa4 & PAMb2 & PAMb1 \\
\hline & & PAMa6 & PAMb3 & \\
\hline \multirow[t]{2}{*}{6} & PAMa3 & PAMa5 & PAMb1 & PAMb2 \\
\hline & PAMa4 & & PAMb3 & \\
\hline \multirow[t]{2}{*}{7} & PAMa5 & PAMa6 & PAMb1 & PAMb3 \\
\hline & & & PAMb2 & \\
\hline \multirow[t]{2}{*}{8} & PAMa5 & PAMa4 & PAMb1 & PAMb3 \\
\hline & & PAMa6 & PAMb3 & \\
\hline \multirow[t]{2}{*}{9} & PAMa3 & PAMa5 & PAMb1 & PAMb3 \\
\hline & PAMa4 & PAMa6 & PAMb2 & PAMb4 \\
\hline \multirow[t]{3}{*}{10} & PAMa3 & PAMa4 & PAMb1 & \\
\hline & & PAMa5 & PAMb2 & \\
\hline & & & PAMb3 & \\
\hline \multirow[t]{3}{*}{11} & PAMa5 & PAMa3 & PAMb1 & PAMb4 \\
\hline & & & PAMb2 & \\
\hline & & & PAMb3 & \\
\hline \multirow[t]{2}{*}{12} & PAMa6 & PAMa5 & PAMb3 & PAMb1 \\
\hline & & & & PAMb2 \\
\hline \multirow[t]{2}{*}{13} & PAMa3 & PAMa4 & PAMb1 & PAMb2 \\
\hline & PAMa6 & & & PAMb3 \\
\hline
\end{tabular}

para los indicadores de efectividad, rentabilidad y utilidad, y de manera ascendente para el indicador de costo unitario. Tanto en la empresa A como en la $B$, se puede observar que la zona 9 es la que se repite y entrega mejor resultado en efectividad, rentabilidad, utilidad y un menor costo unitario, seguidas por las zonas 8,10 y 4 (Tablas 5 y 6).

Determinación de embarcaciones pesqueras más eficientes

Las embarcaciones pesqueras fueron analizadas en cada zona de pesca a través del estadístico $\mathrm{F}$ de Fisher, sin encontrar diferencias significativas en el indicador captura por lance, entre las embarcacio- nes (a un 5\% de error). Habiendo evaluado cada indicador, se estimaron las anomalías de los indicadores tanto de comportamiento como de comparación las que se presentan gráficamente en el plano cartesiano de control. Los gráficos cartesianos de las anomalías de comportamiento versus anomalías de comparación, muestran la ubicación de cada embarcación en un cuadrante determinado para cada zona analizada. De acuerdo con la frecuencia de ocurrencia de cada embarcación en cada cuadrante, se determinaron las embarcaciones con mejores indicadores, y por lo tanto más apropiadas para operar en las zonas de pesca. De los resultados obtenidos, a continuación sólo se presentan los obtenidos en las zonas con mejores índices de eficiencia. En 
Tabla 5. Indicadores para la selección de caladeros por la empresa A.

Table 5. Indicators for the selection of the fishing grounds by company $A$.

\begin{tabular}{cccccccc}
\hline \multicolumn{7}{c}{ Indicadores por zona de pesca } \\
\hline Zona & $\begin{array}{c}\text { Efectividad } \\
\text { (UF) }\end{array}$ & Zona & $\begin{array}{c}\text { Costo Unitario } \\
(\mathbf{U F})\end{array}$ & $\begin{array}{c}\text { Zona } \\
\left(\mathbf{U F} \cdot \mathbf{h}^{-1}\right)\end{array}$ & Rentabilidad $^{-}$ & $\begin{array}{c}\text { Zona } \\
(\mathbf{U F})\end{array}$ & Utilidad \\
\hline $\mathbf{8}$ & $\mathbf{9 9 6 , 5}$ & $\mathbf{9}$ & $\mathbf{8 , 9 0}$ & $\mathbf{8}$ & $\mathbf{1 1 , 9 4}$ & $\mathbf{9}$ & $\mathbf{3 0 8 7 5 7 3}$ \\
10 & 781,2 & 10 & 11,12 & 10 & 11,67 & 10 & 2913099 \\
9 & 526,9 & 8 & 12,71 & 9 & 7,15 & 8 & 1264690 \\
7 & 425,3 & 2 & 12,91 & 6 & 4,86 & 6 & 1188750 \\
6 & 406,2 & 6 & 13,54 & 2 & 4,34 & 2 & 581110 \\
5 & 343,3 & 13 & 15,94 & 13 & 3,79 & 13 & 471631 \\
3 & 341,1 & 7 & 17,45 & 7 & 3,21 & 5 & 329183 \\
13 & 324,9 & 4 & 20,49 & 5 & 2,86 & 7 & 249682 \\
4 & 305,4 & 5 & 22,62 & 4 & 2,85 & 4 & 238935 \\
1 & 263,3 & 12 & 25,8 & 3 & 2,24 & 12 & 175586 \\
12 & 246,5 & 3 & 27,54 & 12 & 2,17 & 3 & 68150 \\
2 & 189,7 & 1 & 32,17 & 1 & 1,74 & 1 & 24077 \\
11 & 188,4 & 11 & 68,52 & 11 & 0,97 & 11 & -6052 \\
\hline
\end{tabular}

Tabla 6. Indicadores para la selección de caladeros por la empresa $B$.

Table 6. Indicators for the selection of the fishing grounds by company $B$.

\begin{tabular}{cccccccc}
\hline \multicolumn{7}{c}{ Indicadores por zona de pesca } \\
\hline Zona & $\begin{array}{c}\text { Efectividad } \\
\text { (UF) }\end{array}$ & Zona & $\begin{array}{c}\text { Costo Unitario } \\
\text { (UF) }\end{array}$ & Zona & $\begin{array}{c}\text { Rentabilidad } \\
\left(\mathbf{U F} \cdot \mathbf{h}^{-1}\right)\end{array}$ & Zona & $\begin{array}{c}\text { Utilidad } \\
(\mathbf{U F})\end{array}$ \\
\hline $\mathbf{9}$ & $\mathbf{6 5 5 , 5}$ & $\mathbf{9}$ & $\mathbf{9 , 3 3}$ & $\mathbf{9}$ & $\mathbf{7 , 8 6}$ & $\mathbf{9}$ & $\mathbf{2 3 0 0 1 0 3}$ \\
4 & 551,8 & 10 & 11,71 & 10 & 6,27 & 10 & 2192990 \\
3 & 547,2 & 2 & 12,17 & 2 & 6,03 & 11 & 1326430 \\
2 & 460,5 & 4 & 14,01 & 4 & 5,24 & 2 & 746294 \\
8 & 406,8 & 3 & 14,33 & 3 & 5,12 & 6 & 670434 \\
10 & 402,6 & 6 & 15,53 & 6 & 4,72 & 3 & 461967 \\
5 & 387,3 & 11 & 15,97 & 11 & 4,59 & 12 & 340394 \\
11 & 360,2 & 13 & 22,38 & 13 & 3,28 & 4 & 309291 \\
6 & 342,2 & 8 & 23,15 & 8 & 3,17 & 8 & 306002 \\
13 & 333,2 & 12 & 24,95 & 12 & 2,94 & 13 & 273895 \\
7 & 303,8 & 5 & 26,95 & 5 & 2,72 & 5 & 147401 \\
12 & 278,2 & 1 & 40,55 & 1 & 1,81 & 1 & 39483 \\
1 & 241,5 & 7 & 62,04 & 7 & 1,18 & 7 & 18484 \\
\hline
\end{tabular}

las Figuras 7 y 8 se presentan los gráficos cartesianos de las anomalías de comportamiento versus las anomalías de comparación de la empresa A en la zona 9 , y de la empresa B en la zona 10 respectivamente.

Para la zona 9, se muestra el comportamiento de cada indicador por embarcación (Fig. 6). Se destaca al PAMa3 (Fig. 6b), que presenta indicadores ubicados en el cuadrante 1 , lo que indica una opera- ción eficiente. Por el contrario, si se observa el PAMa6 (Fig. 6c) al ubicarse en el cuadrante 4 indicaría una operación deficiente.

Así mismo, se indican las anomalías por indicador y PAM para la empresa B (Fig. 7). Se observa con anomalía positiva al PAMb1 que se ubica en el cuadrante 1, y al PAMb3 con anomalía del índice general positiva y negativa del índice de comporta- 
miento, mostrando una operación deficiente. Luego, al jerarquizar las embarcaciones según los indicadores de comportamiento, es posible determinar las embarcaciones más eficientes, por zona de pesca para las empresas A y B, así como las zonas de pesca más eficientes (Tablas 7 y 8).

\section{Análisis comparativo de la utilización histórica de las zonas de pesca y embarcaciones, respecto a la selección de zonas de pesca y embarcaciones}

\section{Análisis comparativo del uso de zonas de pesca}

En el diagnóstico realizado de la operación histórica de pesca de la empresa A, sobre las zonas de pesca, se constató que la zona con mayor frecuencia de visita fue la 9 que a pesar de no entregar buenos resultados en cuanto al rendimiento histórico (debido, tal vez, a una cantidad de biomasa del recurso insuficiente en el área), ha entregado altas capturas por tiempo de arrastre o esfuerzo aplicado siendo, probablemente, una de las razones que ha impulsado a operar en este sector. El presente trabajo, permitió observar que efectivamente se trata de la zona de mayor utilidad y bajo costo unitario seguido por la zona 8, que es la zona con mayor efectividad y rentabilidad. Sin embargo, se debe señalar por un lado, que el indicador de efectividad no considera en su evaluación al costo de operación, y por otro lado el índice de rentabilidad puede entregar una visión errada ya que si los ingresos y costos varían proporcionalmente en el indicador, este se mantie-

Tabla 7. Embarcaciones de uso potencial por la empresa $A$.

Table 7. Vessels with potential use by company A.

\begin{tabular}{ccc}
\hline Zona & $\mathbf{1}^{\text {a }}$ Prioridad & $\mathbf{2}^{\text {a }}$ Prioridad \\
\hline 9 & PAMa3 & PAMa5 \\
8 & PAMa4 & PAMa6 \\
10 & PAMa5 & PAMa3PAMa4 \\
\hline
\end{tabular}

Tabla 8. Embarcaciones de uso potencial por la empresa $B$.

Table 8 . Vessels with potential use by company $B$.

\begin{tabular}{ccc}
\hline Zona & $\mathbf{1}^{\text {a }}$ Prioridad & $\mathbf{2}^{\text {a }}$ Prioridad \\
\hline 9 & PAMb4 & \\
10 & PAMb1PAMb2 & PAMb4 \\
4 & PAMb1PAMb3 & PAMb2 \\
\hline
\end{tabular}

ne constante. Por lo tanto, al considerar al criterio de utilidad como el de selección de la zona de pesca o caladero, se observa a la zona 9 como la más conveniente. Luego, le siguen las zonas 8 y 10, como aquellas con los mejores indicadores de selección.

De acuerdo al análisis realizado en la empresa $\mathrm{B}$, las zonas más visitadas fueron las 10 y 9, mostrando esta última un alto rendimiento de pesca. Por otro lado, en ambas zonas se observó una alta captura por unidad de esfuerzo, pero no así una alta abundancia del recurso. Sin embargo, a pesar de esto último, el estudio demostró que en la zona 9 se observó mayor efectividad, rentabilidad y utilidad, como también un menor costo unitario, seguida posteriormente por la zona 10 en términos de rentabilidad, utilidad y menor costo unitario, y luego la zona 4 según el indicador de efectividad.

\section{Análisis comparativo del uso de embarcaciones pesqueras}

Una vez realizado el diagnóstico de la operación histórica de pesca de ambas empresas, y sobre la base de la evaluación de selección de embarcaciones más eficientes a operar en cada una de las zonas de pesca, se observó que durante 1998-2002 se operó con algunas de las embarcaciones más eficientes, exceptuando algunos casos.

Por un lado, la empresa A (Tablas 4 y 7) particularmente en la zona 9 coincide en prioridad como en embarcación seleccionada. No sucede lo mismo para las zonas 8 y 10 , donde son coincidentes en algunas embarcaciones a utilizar, pero no así en prioridad. La empresa B por otro lado, debiera operar con el PAMb4, sin embargo, operó con el PAMb1 y PAMb2 como primera prioridad, y se consideró al PAMb4 como segunda alternativa de uso. Esto pudo ser por motivos no contemplados en el presente estudio, como fallas de la embarcación, carenas u otros.

En la zona 10, la empresa A operó con mayor frecuencia con el PAMa3, teniendo como segunda opción al PAMa4 y PAMa5. Sin embargo, a través del estudio se descubrió que es este último PAM el que definitivamente entrega un mayor indicador de eficiencia económica. En esta misma zona, la empresa B, opera con el PAMb1, PAMb2 y PAMb3, lo que según este estudio coincide para los dos primeros PAM, como las primeras prioridades, debiendo seguir como segunda prioridad el PAMb4. 

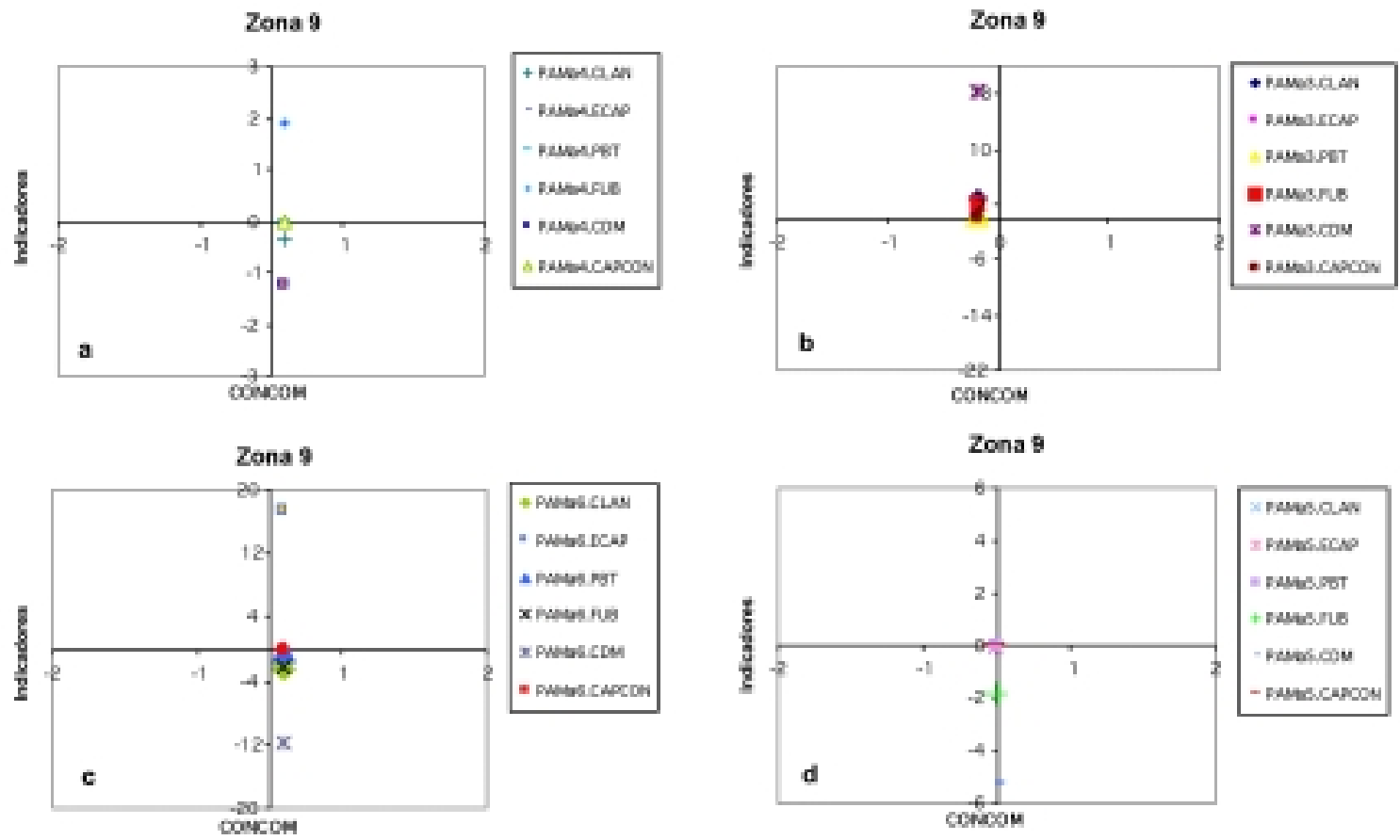

Figura 6. Gráficos Cartesianos de las anomalías de comportamiento versus anomalías de comparación (CONCOM) de la empresa A en la zona 9. a) PAMa4, b) PAMa3, c) PAMa6 y d) PAMa5.

Figure 6. Cartesian graphs of the behavior anomalies versus comparison anomalies (CONCOM) for company $\mathrm{A}$ in zone 9. a) PAMa4, b) PAMa3, c) PAMa6 and d) PAMa5.

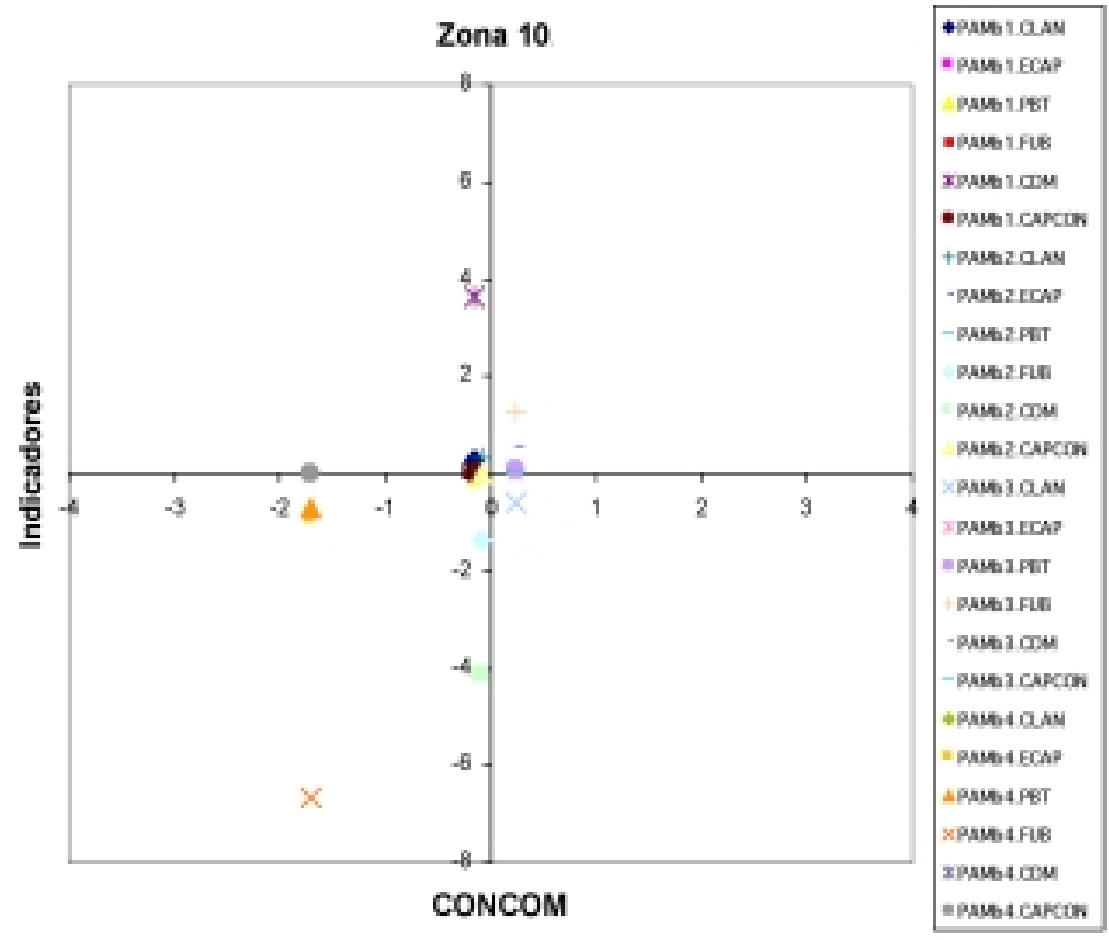

Figura 7. Gráfico cartesiano de las anomalías de comportamiento versus anomalías de comparación de la empresa $B$ en la zona 10.

Figure 7. Cartesian graph of the behavior anomalies versus comparison a n o m a l i e s (CONCOM) for company $B$ in zone 10. 


\section{DISCUSION}

En el presente estudio, se estudia la operación de dos empresas pesqueras industriales de extracción, pertenecientes a la flota de la pesquería de merluza común, entre 1998-2002. Se analizan las zonas de pesca y las embarcaciones que operaron en ellas. Para ello, uno de los aspectos más importantes fue el levantamiento de la información, producto de las limitaciones al acceso y la calidad de los datos disponibles sobre la operación de la flota, dada sus características de información reservada, a pesar que en algunas variables fue posible realizar estimaciones. En efecto, dado que las bitácoras con las que cuenta el INPESCA no indican los datos operativos de la totalidad de la flota, si no más bien de algunas embarcaciones (precisamente las de mayor capacidad de bodega), fue necesario recurrir a información de ambas empresas, la que es escasa y difícil de conseguir, por tratarse de información confidencial relacionada con los costos asociados a ellas.

La utilización de indicadores de desempeño para la evaluación de la operación de la flota, permite representar, de manera simple, las condiciones de operación y evaluar si las zonas de pesca seleccionadas son las adecuadas, y si las embarcaciones utilizadas para la extracción, se han desempeñado de manera eficiente en cada una de ellas. Estos indicadores son similares a los sugeridos por la FAO (2001), para el análisis de la pesca de extracción. Sin embargo, la literatura sobre evaluación de flotas pesqueras se orienta a nivel de análisis sobre una pesquería en que están asociadas muchas empresas $\mathrm{y}$ una unidad de stock del recurso objetivo (Rose $e t$ al., 2003; Peña et al., 2003). En este sentido, los estudios se enfocan a una evaluación de la pesquería más que a la operación de la flota y la selección de zonas de pesca, de manera de detectar operaciones de pesca eficientes y mejorar el uso de los recursos con que dispone la empresa.

El método de jerarquización de la flota, ha sido utilizado en el análisis de la flota de cerco industrial en la zona norte de Chile y en la zona centro-sur. Sin embargo, en las pesquerías demersales no se ha observado la aplicación de este tipo de análisis. Este método puede ser utilizado siempre que se disponga de una base de datos, que permita la evaluación de los indicadores mencionados. Además, es posible mejorar el método incorporando estadística no paramétrica o bien desarrollando un método de normalización de los indicadores, de manera de generar un ranking de zonas de pesca o caladeros.
En relación a los resultados obtenidos en el presente trabajo, se debe destacar que corresponde a una fracción de la flota pesquera industrial que opera sobre la pesquería de merluza común. Adicionalmente, el análisis ha sido enfocado a la captura de esta especie sin considerar en el análisis de eficiencia, la presencia de fauna acompañante y su aporte a la operación de pesca. Lo anterior, puede corresponder a futuros análisis y a trabajos similares a éste.

Si se considera que el estudio comprende el periodo 1998-2002, se debe tener presente que se observa un primer período correspondiente a la etapa previa a la aplicación de la medida de administración de Límite Máxima de Captura por Armador (LMCA) entre 1998-2001, y otro período inmediatamente posterior a la aplicación del LMCA, en 2002. En este sentido, el presente trabajo no se orientó a observar si existieron cambios de estrategias operacionales de la flota pesquera en ambas empresas, que determinaran las zonas de pesca y embarcaciones a utilizar. Esta problemática, es una extensión interesante a desarrollar a partir de este estudio, incorporando los efectos de las medidas de administración en la selección de zonas de pesca y eficiencia de operación de los pesqueros de alta mar.

Con respecto a las empresas analizadas, se puede indicar que se han desempeñado de manera eficiente y por ende han sido asertivas, lo que se debería a la experiencia adquirida a través de los años, por quienes toman las decisiones sobre la selección de las zonas de pesca y de las embarcaciones a operar en ellas.

En el análisis de las embarcaciones más utilizadas, las empresas A y B presentan un cambio continuo en su uso, que puede ser debido a que en ocasiones las embarcaciones se encuentran en mantención, reparación, carena, diques o bien destinadas a la pesca de otro recurso.

Los planos cartesianos de ordenamiento de las anomalías por cuadrante propuestos en este trabajo, son de fácil comprensión y manejo. A partir de estas gráficas, y considerando que cada cuadrante lleva asociado un criterio de eficiencia, es posible jerarquizar la flota pesquera.

Los resultados obtenidos, considerando los datos históricos analizados para dos empresas pesqueras, durante el período 1998-2002, permiten caracterizar por medio de indicadores, qué zonas de pesca resultan ser las más eficientes económicamente para dirigir los esfuerzos pesqueros. Además, el análisis histórico permite identificar qué embarcaciones fueron 
las más eficientes, para cada zona analizada. Considerando ambos resultados, es posible señalar a cada empresa hacia qué zona de pesca dirigir sus esfuerzos y con qué embarcación. Posteriormente, al comprar estos resultados de selección de zonas de pesca y embarcaciones, respecto al comportamiento histórico de cada empresa en la selección de zonas de pesca y embarcaciones, es posible indicar que éstas presentaron comportamientos cercanos a los criterios de selección. Si la toma de decisiones de zonas de pesca es efectuada por los propios capitanes de las embarcaciones, se puede pensar que ellos poseen ciertos antecedentes, como información del comportamiento del recurso, que les permite ser asertivos en su selección. Sin embargo, al observar que existen ciertas zonas donde han operado embarcaciones que no serían las más eficientes, permite indicar que una herramienta objetiva, como la planteada en el presente estudio, sería útil para efectos de orientación tanto a capitanes como a los jefes de flota de las empresas pesqueras industriales.

\section{AGRADECIMIENTOS}

Los autores agradecen las facilidades otorgadas por el Instituto de Investigación Pesquera (INPESCA) y a las empresas pesqueras involucradas en el estudio, quienes aportaron los antecedentes necesarios para el desarrollo de este trabajo. Se agradece al Sr. Luis Cubillos, por la valiosa colaboración y aportes que contribuyeron a enriquecer este trabajo. Adicionalmente, deseamos agradecer a los evaluadores anónimos de este trabajo, por los aportes entregados.

\section{REFERENCIAS}

Burgos, G. 2004. Análisis comparativo de la utilización actual de los caladeros de pesca y embarcaciones de merluza común (Merluccius gayi gayi), respecto al uso potencial por parte de las empresas pesqueras de la VIII Región. Tesis de Ingeniería Pesquera, Universidad Católica de la Santísima Concepción, 150 pp.

Canavos, G. 1998. Probabilidad y estadística. Aplicaciones y métodos. McGraw-Hill, México, 651 pp.

Cerda, D. 2001. Eficiencia del uso de combustible de la flota cerquera industrial de la zona centro-sur de Chile durante 1999. Tesis de Ingeniería Pesquera, Pontificia Universidad Católica de Valparaíso, $130 \mathrm{pp}$.

Díaz, C. 1993. Mecanismo de control de una flota cerquera industrial de la zona norte de Chile. Tesis de Ingeniería Pesquera, Pontificia Universidad Católica de Valparaíso, 145 pp.

Gatica, C. \& L. Cubillos. 2004. Análisis de la situación de stock de merluza común entre 1992 y 2003. Doc. Tec. INPESCA, Talcahuano, 12(19):1-23 pp.

Instituto de Fomento Pesquero (IFOP). 2000. Investigación de la situación pesquera demersal zona centro-sur 2000. Inf. Téc., IFOP, Valparaíso, 120 pp.

Instituto de Investigación Pesquera (INPESCA). 2002. Situación de la merluza común 2002 y captura permisible 2003. Inf. Téc., INPESCA, Talcahuano, $10 \mathrm{pp}$.

Olivares, J. 1977. Consideraciones generales en la evaluación de una flota pesquera. Tesis de Técnico Pesquero, Universidad Católica de Valparaíso, 87 pp.

Organización de Naciones Unidas para la Agricultura y la Alimentación (FAO). 2001. Directrices para la recopilación sistemática de datos relativos a la pesca de captura. Doc. Tec. Pesca, 382: 111 pp.

Peña, J., M. Basch \& S. Vergara. 2003. Eficiencia técnica y escalas de operación en pesca pelágica: un análisis de fronteras estocásticas. Cuad. Econ., 40(119): 47-87.

Rebolledo, H., C. Gatica \& L. Cubillos. 2002. Aspectos operacionales de la pesquería industrial de merluza común (Merluccius gayi), 1997-2002. Doc. Téc. INPESCA, Talcahuano, 11(5): 1-23.

Rose, R., M. Stubbs, P. Gooday \& A. Cox. 2003. Economic performance indicators for fisheries. Australian Bureau of Agricultural and Resources Economics, Australia, 10 pp.

Salas, N., T. Melo, F. Allaami \& P. Reyes. 1985. Poder de pesca funcional de embarcaciones de cerco. En: T. Melo (ed.). Estudios en pesquerías chilenas. Escuela de Ciencias del Mar, Universidad Católica de Valparaíso, pp. 123-130. 
Investigaciones Marinas, Vol. 33(2) 2005 